\title{
Stability of Excited Atoms in Small Cavities
}

\author{
G. Flores-Hidalgo *, A.P.C. Malbouisson ${ }^{\dagger}$ and Y.W. Milla ${ }^{\ddagger}$ \\ Centro Brasileiro de Pesquisas Físicas, Rua Dr. Xavier Sigaud 150, Urca, Rio de Janeiro CEP 22290-180-RJ, Brazil.
}

\begin{abstract}
We consider a system consisting of an atom in the approximation of a harmonic oscillator of frequency $\bar{\omega}$, coupled to the scalar potential inside a spherical reflecting cavity of radius $R$. We use dressed states introduced in a previous publication [Andion, Malbouisson, and Mattos Neto, J. Phys. A 34, 3735 (2001)], which allow a non-perturbative unified description of the atom radiation process, in both cases, of a finite or an arbitrarily large cavity. We perform a study of the energy distribution in a small cavity, with the initial condition that the atom is in the first excited state and we conclude for the quasi-stability of the excited atom. For instance, for a frequency $\bar{\omega}$ of the order $\bar{\omega} \sim 4.00 \times 10^{14} / \mathrm{s}$ (in the visible red), starting from the initial condition that the atom is in the first excited level, we find that for a cavity with diameter $2 R \sim 1.0 \times 10^{-6} \mathrm{~m}$, the probability that the atom be at any time still in the first excited level, will be of the order of $97 \%$. For a typical microwave frequency $\bar{\omega} \sim 2,00 \times 10^{10} / s$ we find stability in the first excited state also of the order of $97 \%$ for a cavity radius $R \sim 1.4 \times 10^{-2} \mathrm{~m}$.
\end{abstract}

PACS Number(s): 03.65.Ca, 32.80.Pj

\section{INTRODUCTION}

There are situations in the domain of Atomic Physics, Cavity Electrodynamics and Quantum Optics, where perturbation methods are of little usefulness, for instance, in resonant effects associated to the coupling of atoms with strong radiofrequency fields [1]. The theoretical understanding of these effects on perturbative grounds requires the calculation of very high-order terms in perturbation series, what makes the standard Feynman diagrams technique practically unreliable in those cases [1]. The trials of treating non-perturbativelly such kind of systems, have lead to the idea of dressed atom, introduced in refs [2] and [3]. Since then this concept has been used to investigate several situations involving the interaction of atoms and electromagnetic fields ( [4], [5], [6]). A way to circumvect the mathematical difficulties due to non-linear character of the problem, is to assume that under certain conditions the coupled atom-electromagnetic field system may be approximated by the system composed of an harmonic oscillator coupled linearly to the field trough some effective coupling constant $g$. This is the case in the context of the general $Q E D$ linear response theory, where the electric dipole interaction gives the leading contribution to the radiation process ( [7], [8], [9], [10]) and also in several branches of Quantum Optics ( [11], [12], [13]).

We consider a system composed of an atom (approximated by a harmonic oscillator) coupled linearly to the scalar potential, the whole system being confined inside a reflecting sphere of radius $R$. We give a non-perturbative treatment to the field-atom system introducing some dressed coordinates that allow to divide the coupled system into two parts, the dressed atom and the dressed field, what makes unnecessary to work directly with the concepts of bare atom, bare field and interaction between them. For instance, to describe the radiation process, having as initial condition that only the mechanical oscillator (the atom), $q_{0}$ be excited, the usual procedure is to consider the interaction term in the Hamiltonian written in terms of $q_{0}$ and the field modes $q_{i}$ as a perturbation, which induces transitions among the eigenstates of the free Hamiltonian. In this way it is possible to treat approximately the problem having as initial condition that only the bare mechanical oscillator (the atom) be excited. But as is well known this initial condition is physically not consistent in reason of the divergence of the bare oscillator frequency, due to the interaction with the field. The traditional way to circumvect this difficulty is by the renormalization procedure, introducing perturbativelly order by order corrections to the oscillator frequency. In this paper we adopt an alternative procedure introduced in [14. We do not make explicit use of the concepts of interacting bare oscillator and field, described by the coordinates $q_{0}$ and $\left\{q_{i}\right\}$. We introduce dressed coordinates $q_{0}^{\prime}$ and $\left\{q_{i}^{\prime}\right\}$ for, respectively the dressed atom and the dressed field modes. In terms of these new coordinates a non-perturbative approach of the radiation process and of the distribution of energy inside the cavity is possible.

\footnotetext{
*E-mail: gflores@cbpf.br

${ }^{\dagger}$ E-mail: adolfo@cbpf.br

${ }^{\ddagger}$ E-mail: yonym@cbpf.br
} 
We start considering an atom approximated by a harmonic oscillator $q_{0}(t)$ of frequency $\omega_{0}$ (we will introduce below a renormalized frequency $\bar{\omega}$ which is physically meaningfull) coupled linearly to the scalar potential $\phi$, the whole system being confined in a sphere of radius $R$ centered at the oscillator position. The equations of motion are,

$$
\begin{gathered}
\ddot{\mathrm{q}}_{0}(t)+\omega_{0}^{2} q_{0}(t)=2 \pi \sqrt{g c} \int_{0}^{R} d^{3} \mathbf{r} \phi(\mathbf{r}, t) \delta(\mathbf{r}), \\
\frac{1}{c^{2}} \frac{\partial^{2} \phi}{\partial t^{2}}-\nabla^{2} \phi(\mathbf{r}, t)=2 \pi \sqrt{g c} q_{0}(t) \delta(\mathbf{r}) .
\end{gathered}
$$

Using a basis of spherically symmetric Bessel functions defined in the domain $0<|\mathbf{r}|<R$, the equations above can be written as a set of equations coupling the atom to the harmonic field modes, which can be derived from the Hamiltonian

$$
H=\frac{1}{2}\left[p_{0}^{2}+\omega_{0}^{2} q_{0}^{2}+\sum_{k=1}^{N}\left(p_{k}^{2}+\omega_{k}^{2} q_{k}^{2}-2 \eta \omega_{k} q_{0} q_{k}\right)\right] .
$$

\section{THE EIGENFREQUENCIES SPECTRUM}

We consider for a moment as in [14], the problem of a harmonic oscillator $q_{0}$ coupled to $N$ other oscillators. In the limit $N \rightarrow \infty$ we recover our original situation of the coupling oscillator-field after redefinition of divergent quantities, in a manner analogous as renormalization is done in field theories. In the above equations, $g$ is a coupling constant (with dimension of frequency), $\eta=\sqrt{2 g \Delta \omega}$ and $\Delta \omega=\pi c / R$ is the interval between two neighbouring field frequencies, $\omega_{i+1}-\omega_{i}=\Delta \omega=\pi c / R$ and $q_{i}$ stands for the harmonic modes of the field. The Hamiltonian (1.3) can be turned to principal axis by means of a point transformation, $q_{\mu}=t_{\mu}^{r} Q_{r}, p_{\mu}=t_{\mu}^{r} P_{r}$, performed by an orthonormal matrix $T=\left(t_{\mu}^{r}\right), \quad \mu=(0, k), \quad k=1,2, \ldots N, r=0, \ldots N$. The subscript 0 and $k$ refer respectively to the atom and the harmonic modes of the field and $r$ refers to the normal modes. The transformed Hamiltonian in principal axis reads, $H=\frac{1}{2} \sum_{r=0}^{N}\left(P_{r}^{2}+\Omega_{r}^{2} Q_{r}^{2}\right)$, where the $\Omega_{r}$ 's are the normal frequencies corresponding to the possible collective oscillation modes of the coupled system. The eigenfrequencies $\Omega_{r}$ satisfy the equation [14],

$$
\omega_{0}^{2}-N \eta^{2}-\Omega^{2}=\eta^{2} \sum_{k=1}^{N} \frac{\Omega^{2}}{\omega_{k}^{2}-\Omega^{2}} .
$$

The $N+1$ solutions $\Omega_{r}$ of Eq.(2.1), correspond to the $N+1$ normal collective oscillation modes.

It can be shown [14] that if $\omega_{0}^{2}>N \eta^{2}$, Eq. 2.1) yields only positive solutions for $\Omega^{2}$ (all collective modes are harmonic), while if $\omega_{0}^{2}<N \eta^{2}$, Eq.(2.1) has a negative solution $\Omega_{-}^{2}$. This means that there is a damped oscillation mode that does not allows stationary configurations. We will not care about this last situation. Nevertheless it should be remarked that in a different context, it is precisely this negative squared frequency solution (runaway solution) that is related to the existence of a bound state in the Lee-Friedrechs model. This solution is considered in ref. 15] in the framework of a model to describe qualitatively the existence of bound states in particle physics. Thus we take $\omega_{0}^{2}>N \eta^{2}$ and define the renormalized oscillator frequency $\bar{\omega}, \bar{\omega}=\sqrt{\omega_{0}^{2}-N \eta^{2}}$. In the limit $N \rightarrow \infty$ the meaning of the frequency renormalization becomes clear. It is exactly the analogous of a mass renormalization in field theory, the infinite $\omega_{0}$ being chosen in such a way as to make the renormalized frequency $\bar{\omega}$ finite and equal to the observed oscillator frequency. In terms of the renormalized frequency Eq.(2.1) can be writen, after some manipulations, in the form [14],

$$
\cot \left(\frac{\mathrm{R} \Omega}{\mathrm{c}}\right)=\frac{\Omega}{\pi \mathrm{g}}+\frac{\mathrm{c}}{\mathrm{R} \Omega}\left(1-\frac{\bar{\omega}^{2} \mathrm{R}}{\pi \mathrm{gc}}\right) .
$$

The solutions of Eq.(2.2) with respect to $\Omega$ give the spectrum of eigenfrequencies $\Omega_{r}$ corresponding to the collective normal modes. The transformation matrix elements turning the oscillator-field system to principal axis is obtained taking the limit $N \rightarrow \infty$, after some rather long but straightforward manipulations in [14]. They read,

$$
\begin{aligned}
t_{0}^{r} & =\frac{\Omega_{r}}{\sqrt{\frac{R}{2 \pi g c}\left(\Omega_{r}^{2}-\bar{\omega}^{2}\right)^{2}+\frac{1}{2}\left(3 \Omega_{r}^{2}-\bar{\omega}^{2}\right)+\frac{\pi g R}{2 c} \Omega_{r}^{2}}}, \\
t_{k}^{r} & =\frac{\eta \omega_{k}}{\omega_{k}^{2}-\Omega_{r}^{2}} t_{0}^{r} .
\end{aligned}
$$


We define below some coordinates $q_{0}^{\prime}, q_{i}^{\prime}$ associated to the dressed atom and the dressed field. These coordinates will reveal themselves to be appropriate to give an appealing non-perturbative description of the atom-field system.

\section{DRESSED STATES}

We start from the eigenstates of our system, represented by the normalized eigenfunctions,

$$
\phi_{n_{0} n_{1} n_{2} \ldots}(Q, t)=\prod_{s}\left[\sqrt{\frac{2^{n_{s}}}{n_{s} !}} H_{n_{s}}\left(\sqrt{\frac{\Omega_{s}}{\hbar}} Q_{s}\right)\right] \Gamma_{0} e^{-i \sum_{s} n_{s} \Omega_{s} t},
$$

where $H_{n_{s}}$ stands for the $n_{s}$ th Hermite polynomial and $\Gamma_{0}$ is the normalized vacuum eigenfunction. Let us introduce dressed coordinates $q_{0}^{\prime}$ and $\left\{q_{i}^{\prime}\right\}$ for, respectively the dressed atom and the dressed field, defined by [14],

$$
\sqrt{\frac{\bar{\omega}_{\mu}}{\hbar}} q_{\mu}^{\prime}=\sum_{r} t_{\mu}^{r} \sqrt{\frac{\Omega_{r}}{\hbar}} Q_{r}
$$

valid for arbitrary $R$ and where $\bar{\omega}_{\mu}=\left\{\bar{\omega}, \omega_{i}\right\}$. In terms of the bare coordinates the dressed coordinates are expressed as,

$$
q_{\mu}^{\prime}=\sum_{\nu} \alpha_{\mu \nu} q_{\nu} ; \quad \alpha_{\mu \nu}=\frac{1}{\sqrt{\omega_{\mu}}} \sum_{r} t_{\mu}^{r} t_{\nu}^{r} \sqrt{\Omega_{r}}
$$

Let us define for a fixed instant the complete orthonormal set of functions [14],

$$
\psi_{\kappa_{0} \kappa_{1} \ldots}\left(q^{\prime}\right)=\prod_{\mu}\left[\sqrt{\frac{2^{\kappa_{\mu}}}{\kappa_{\mu} !}} H_{\kappa_{\mu}}\left(\sqrt{\frac{\bar{\omega}_{\mu}}{\hbar}} q_{\mu}^{\prime}\right)\right] \Gamma_{0},
$$

where $q_{\mu}^{\prime}=q_{0}^{\prime}, q_{i}^{\prime}, \bar{\omega}_{\mu}=\left\{\bar{\omega}, \omega_{i}\right\}$. Note that the ground state $\Gamma_{0}$ in the above equation is the same as in Eq.(3.1). The invariance of the ground state is due to our definition of dressed coordinates given by Eq.(3.2). Each function $\psi_{\kappa_{0} \kappa_{1} \ldots}\left(q^{\prime}\right)$ describes a state in which the dressed oscillator $q_{\mu}^{\prime}$ is in its $\kappa_{\mu}-t h$ excited state. Using Eq.(3.2) the functions (3.4) can be expressed in terms of the normal coordinates $Q_{r}$. But since (3.1) is a complete set of orthonormal functions, the functions (3.4) may be written as linear combinations of the eigenfunctions of the coupled system (we take $t=0$ for the moment),

$$
\psi_{\kappa_{0} \kappa_{1} \ldots}\left(q^{\prime}\right)=\sum_{n_{0} n_{1} \ldots} T_{\kappa_{0} \kappa_{1} \ldots}^{n_{0} n_{1} \ldots}(0) \phi_{n_{0} n_{1} n_{2} \ldots}(Q, 0)
$$

where the coefficients are given by,

$$
T_{\kappa_{0} \kappa_{1} \ldots}^{n_{0} n_{1} \ldots}(0)=\int d Q \psi_{\kappa_{0} \kappa_{1} \ldots} \phi_{n_{0} n_{1} n_{2} \ldots}
$$

the integral extending over the whole $Q$-space.

We consider the particular configuration $\psi$ in which only one dressed oscillator $q_{\mu}^{\prime}$ is in its $N$-th excited state, all other being in the ground state,

$$
\psi_{0 \ldots N(\mu) 0 \ldots}\left(q^{\prime}\right)=\left(2^{-N} N !\right)^{-\frac{1}{2}} H_{N}\left(\sqrt{\frac{\bar{\omega}_{\mu}}{\hbar}} q_{\mu}^{\prime}\right) \Gamma_{0} .
$$

The coefficients (3.6) have been calculated in ref. 14]. We get,

$$
T_{0 \ldots N(\mu) 0 \ldots}^{n_{0} n_{1} \ldots}=\left(\frac{N !}{n_{0} ! n_{1} ! \ldots}\right)^{\frac{1}{2}}\left(t_{\mu}^{0}\right)^{n_{0}}\left(t_{\mu}^{1}\right)^{n_{1}} \ldots,
$$

where the subscripts $\mu=0, i$ refer respectively to the dressed atom and the harmonic modes of the field and the quantum numbers satisfy the constraint $n_{0}+n_{1}+\ldots=N$. In the following we focus our attention on the behaviour of the system with the initial condition that only one dressed oscillator $q_{\mu}^{\prime}$ (the dressed atom or one of the modes of the 
dressed field) be in the $N$-th excited state. We will study in detail the particular case $N=1$, which will be enough to have a clear understanding of our approach. Let us call $\Gamma_{1}^{\mu}$ the configuration in which only the dressed oscillator $q_{\mu}^{\prime}$ is in the first excited level. We have from Eqs.(3.7), (3.5) (3.8) and (3.2) the following expression for the time evolution of the first-level excited dressed oscillator $q_{\mu}^{\prime}$,

$$
\Gamma_{1}^{\mu}(t)=\sum_{\nu} f^{\mu \nu}(t) \Gamma_{1}^{\nu}(0)
$$

where the coefficients $f^{\mu \nu}(t)$ are given by

$$
f^{\mu \nu}(t)=\sum_{s} t_{\mu}^{s} t_{\nu}^{s} e^{-i \Omega_{s} t}
$$

From Eq.(3.9) we see that the initially excited dressed oscillator naturally distributes its energy among itself and all other dressed oscillators as time goes on, with probability amplitudes given by Eq.(3.10). If the dressed oscillator $q_{0}^{\prime}$ (the atom) is in its first excited state at $t=0$, its decay rate may evaluated from the time evolution equation,

$$
\Gamma_{1}^{0}(t)=\sum_{\nu} f^{0 \nu}(t) \Gamma_{1}^{\nu}(0)
$$

In Eq. 3.11 the coefficients $f^{0 \nu}(t)$ have a simple interpretation: $f^{00}(t)$ and $f^{0 i}(t)$ are respectively the probability amplitudes that at time $t$ the dressed atom still be excited or have radiated a photon of frequency $\omega_{i}$. We see that this formalism allows a quite natural description of the radiation process as a simple exact time evolution of the system. We consider in the following the time evolution of the excited atom, in the cases of a very large and a very small cavity.

A very large cavity:

In the case of a very large cavity our method generalizes what can be obtained from perturbation theory. The probability that the atom be still excited at time $t$ can be obtained in continuous language from the amplitude given by Eq. (3.10),

$$
f^{00}(t)=\int_{0}^{\infty} \frac{2 g \Omega^{2} e^{-i \Omega t} d \Omega}{\left(\Omega^{2}-\bar{\omega}^{2}\right)^{2}+\pi^{2} g^{2} \Omega^{2}}
$$

For large $t\left(t>>\frac{1}{\bar{\omega}}\right)$, but for in principle arbitrary coupling $g$, we obtain for the probability of finding the atom still excited at time $t$, the result [14],

$$
\left|f^{00}(t)\right|^{2}=e^{-\pi g t}\left(1+\frac{\pi^{2} g^{2}}{4 \tilde{\bar{\omega}}^{2}}\right)-e^{-\pi g t / 2} \frac{8 g}{\bar{\omega}^{4} t^{3}}\left(\sin \tilde{\tilde{\omega}} t+\frac{\pi g}{2 \tilde{\bar{\omega}}} \cos \tilde{\tilde{\omega}} t\right)+\frac{16 g^{2}}{\overline{\bar{\omega}}^{8} t^{6}}
$$

where $\tilde{\bar{\omega}}=\sqrt{\bar{\omega}^{2}-\frac{\pi^{2} g^{2}}{4}}$. In the above expression the approximation $t>>\frac{1}{\bar{\omega}}$ plays a role only in the two last terms, due to difficulties to evaluate exactly the integral in Eq. (3.12) along the imaginary axis using Cauchy's theorem. The first term comes from the residue at $\Omega=\tilde{\tilde{\omega}}+i \frac{\pi g}{2}$ and would be the same if we have done an exact calculation. If we consider in eq. (3.13) $g<<\bar{\omega}$, which corresponds in electromagnetic theory to the fact that the fine structure constant $\alpha$ is small compared to unity (for explicit calculations we take below $g / \bar{\omega}=\alpha$ ), we obtain the well known perturbative exponential decay law.

\section{THE RADIATION PROCESS IN A SMALL CAVITY}

Let us now consider the atom placed at the center of a very small cavity, i.e. that satisfies the condition that its radius be much smaller than the coherence lenght, $R<<c / g$. To obtain the eigenfrequencies spectrum, we remark that from a numerical analysis of Eq.(2.2) it can be seen that in the case of a small cavity radius $R$, its solutions are near the frequency values corresponding to the asymptots of the $\operatorname{curve} \cot \left(\frac{\mathrm{R} \Omega}{\mathrm{c}}\right)$, which correspond to the field modes $\omega_{i}=i \pi c / R$. The smallest solution departs more from the first asymptot than the other larger solutions depart from their respective nearest asymptot. As we take larger and larger solutions, they are nearer and nearer to the values corresponding to the asymptots. For instance, for a cavity radius $R$ of the order of $10^{-2} \mathrm{~m}$ and $\bar{\omega} \sim 10^{10} / \mathrm{s}$, only the lowest eigenfrequency $\Omega_{0}$ is signicantly different from the field frequency corresponding to the first asymptot, all the 
other eigenfrequencies $\Omega_{k}, k=1,2, \ldots$ being very close to the field modes $k \pi c / R$. For higher values of $\bar{\omega}$ (and lower values of $R$ ) the differences between the eigenfrequencies and the field modes frequencies are still smaller.

Thus to solve Eq.(2.2) for the larger eigenfrequencies we expand the function $\cot \left(\frac{\mathrm{R} \Omega}{\mathrm{c}}\right)$ around the values corresponding to the asymptots. We write,

$$
\Omega_{k}=\frac{\pi c}{R}\left(k+\epsilon_{k}\right), \quad k=1,2, . .
$$

with $0<\epsilon_{k}<1$, satisfying the equation,

$$
\cot \left(\pi \epsilon_{\mathrm{k}}\right)=\frac{\mathrm{c}}{\mathrm{gR}}\left(\mathrm{k}+\epsilon_{\mathrm{k}}\right)+\frac{1}{\left(\mathrm{k}+\epsilon_{\mathrm{k}}\right)}\left(1-\frac{\bar{\omega}^{2} \mathrm{R}}{\pi \mathrm{gc}}\right) .
$$

But since for a small cavity every $\epsilon_{k}$ is much smaller than 1, Eq.(4.2) may be linearized in $\epsilon_{k}$, giving,

$$
\epsilon_{k}=\frac{\pi g c R k}{\pi^{2} c^{2} k^{2}-\bar{\omega}^{2} R^{2}} .
$$

Eqs.(4.1) and (4.3) give approximate solutions to the eigenfrequencies $\Omega_{k}, \quad k=1,2 \ldots$

To solve Eq.(2.2) with respect to the lowest eigenfrequency $\Omega_{0}$, let us assume that it satisfies the condition $\Omega_{0} R / c<<$ 1 (we will see below that this condition is compatible with the condition of a small cavity as defined above). Inserting the condition $\Omega_{0} R / c<<1$ in Eq.(2.2) and keeping up to quadratic terms in $\Omega$ the solution for the lowest eigenfrequency $\Omega_{0}$ can be writen,

$$
\Omega_{0}=\frac{\bar{\omega}}{\sqrt{1+\frac{\pi g R}{c}}} .
$$

Consistency between Eq.(4.4) and the condition $\Omega_{0} R / c<<1$ gives a condition on the cavity radius,

$$
R \ll \frac{c}{g} \frac{\pi}{2}\left(\frac{g}{\bar{\omega}}\right)^{2}\left(1+\sqrt{1+\frac{4}{\pi^{2}}\left(\frac{\bar{\omega}}{g}\right)^{2}}\right) .
$$

Let us define the coupling constant $g$ to be such that $g=\bar{\omega} \alpha$, where $\alpha$ is the fine structure constant, $\alpha=1 / 137$. Then the factor multiplying $c / g$ in the above equation is $\sim 0.07$ and the condition $R \ll c / g$ is replaced by a more restrictive assumption $R \ll 0.07(c / g)$. For a typical infrared frequency, for instance $\bar{\omega} \sim 2,0 \times 10^{11} / \mathrm{s}$, our calculations are valid for a radius $R \ll 10^{-3} \mathrm{~m}$.

From Eq.(2.3) and using the above expressions for the eigenfrequencies in a small cavity, we obtain the matrix elements,

$$
\left(t_{0}^{0}\right)^{2} \approx 1-\frac{\pi g R}{c} ; \quad\left(t_{0}^{k}\right)^{2} \approx \frac{2 g R}{\pi c k^{2}} .
$$

To obtain the above equations we have neglected the corrective term $\epsilon_{k}$, from the expressions for the eigenfrequencies $\Omega_{k}$. Nevertheless, corrections in $\epsilon_{k}$ should be included in the expressions for the matrix elements $t_{k}^{k}$, in order to avoid spurious singularities due to our approximation.

Let us consider the situation where the dressed atom is initially in its first excited level. Then from Eq.(3.10) we obtain the probability that it will still be excited after a ellapsed time $t$,

$$
\left|f^{00}(t)\right|^{2}=\left(t_{0}^{0}\right)^{4}+2 \sum_{k=1}^{\infty}\left(t_{0}^{0}\right)^{2}\left(t_{0}^{k}\right)^{2} \cos \left(\Omega_{k}-\Omega_{0}\right) t+\sum_{k, l=1}^{\infty}\left(t_{0}^{k}\right)^{2}\left(t_{0}^{l}\right)^{2} \cos \left(\Omega_{k}-\Omega_{l}\right) t .
$$

Using Eqs.(4.6) in Eq.(4.7), we obtain

$$
\left|f^{00}(t)\right|^{2}=1-\pi \delta+4\left(\frac{\delta}{\pi}-\delta^{2}\right) \sum_{k=1}^{\infty} \frac{1}{k^{2}} \cos \left(\Omega_{k}-\Omega_{0}\right) t \pi^{2} \delta^{2}+\frac{4}{\pi^{2}} \delta^{2} \sum_{k, l=1}^{\infty} \frac{1}{k^{2} l^{2}} \cos \left(\Omega_{k}-\Omega_{l}\right) t,
$$

where we have introduced the adimensional parameter $\delta=R g / c \ll 1$, corresponding to a small cavity and we remember that the eigenfrequencies are given by Eqs.(4.1) and (4.3). As time goes on, the probability that the atom be excited attains periodically a minimum value which has a lower bound given by, 


$$
\operatorname{Min}\left(\left|\mathrm{f}^{00}(\mathrm{t})\right|^{2}\right)=1-\frac{5 \pi}{3} \delta+\frac{14 \pi^{2}}{9} \delta^{2} .
$$

For a frequency $\bar{\omega}$ of the order $\bar{\omega} \sim 4.00 \times 10^{14} / \mathrm{s}$ (in the red visible), which corresponds to $\delta \sim 0.005$ and $2 R \sim$ $1.0 \times 10^{-6} \mathrm{~m}$, we see from Eq. (4.9) that the probability that the atom be at any time excited will never fall below a value $\sim 0.97$, or a decay probability that is never higher that a value $\sim 0.03$. In other words, atoms having such emission frequency, placed in a such a small cavity in the first excited level, will be stable in the excited state to the order of $97 \%$. It is interesting to compare this result with experimental observations in [16], [17], where stability is found for atoms emiting in the visible range placed between two parallel mirrors a distance $L=1.1 \times 10^{-6} \mathrm{~m}$ apart from one another. For lower frequencies the size of the cavity ensuring quasi-stability of the same order as above, for the excited atom may be considerably larger. For instance, for $\bar{\omega}$ in a typical microwave value, $\bar{\omega} \sim 2,00 \times 10^{10} / s$ and taking also $\delta \sim 0.005$, the probability that the atom remain in the first excited level at any time will be larger than a value of the order of $97 \%$, for a cavity radius $R \sim 1.0 \times 10^{-2} \mathrm{~m}$. The probability that the atom remain excited as time goes on oscillates with time between a maximum and a minimum values and never departs significantly from the situation of stability of the atom in the excited state. Indeed for an emission frequency $\bar{\omega} \sim 4.00 \times 10^{14} / s$ (in the red visible) considered above and $R \sim 1.0 \times 10^{-6} \mathrm{~m}$, the period of oscillation between the minimum and maximum values of the probability that the atom be excited, is $T \sim \frac{1}{12} \times 10^{-14} \mathrm{~s}$, while for $\bar{\omega} \sim 2,00 \times 10^{10} / \mathrm{s}$, and $R \sim 1.4 \times 10^{-2} \mathrm{~m}$, the period is $T \sim \frac{1.4}{6} \times 10^{-10} s$.

\section{CONCLUDING REMARKS}

We have used in this paper a formalism that allows an unified approach to the radiation process by an atom, in rather different situations, as the atom confined in a very small cavity or in free space. The behaviour of atoms confined in small cavities is completelly different from the behaviour of an atom in free space or in a large cavity. In the first case the emission process is very sensitive to the presence of boundaries, a fact that has been pointed out since a long time ago in the literature ( [18], [19], 20]). Our dressed states approach gives an unified description for the dressing of the atom by the field modes and the emission process in a cavity of arbitrary size, which includes microcavities and very large cavities (free space emission). We recover here with our formalism the experimental observation that excited states of atoms in sufficiently small cavities are stable. We are able to give formulas for the probability of an atom to remain excited for an infinitely long time, provided it is placed in a cavity of appropriate size. For an emission frequency in the visible red, the size of such cavity is in good agreement with experimental observations ( 16, 117]). Also, our approach gives results in good agreement with previous theoretical results for the emission in free space, generalizing the well known exponential decay law. Moreover the detailed behaviours which we obtain with our formalism are very different in the two situations: The atom in a very large cavity has a probability decay rate weekly oscillating and monotonically varying with time (the probability that the atom be excited decreases almost exponentially with increasing time). In the case of an excited atom placed in the center of a very small cavity, the probability that it remains excited as time goes on oscillates very rapidly with time and never departs significantly

from the situation of stability of the atom in the excited state.

\section{ACKNOWLEDGEMENTS}

This work received financial support from $\mathrm{CNPq}$ (Brazilian National Research Council) and FAPERJ (Foundation for the Support of Research in the State of Rio de Janeiro (Brazil)). One of us (A.P.C.M.) is grateful to C. de Calan (Ecole Polytechnique, Paris), F.S. Nogueira and H. Kleinert (Freie Universität, Berlin) for interesting discussions.

[1] C. Cohen-Tannoudji, Atoms in Electromagnetic Fields, (World Scientific, Singapore, 1994).

[2] N. Polonsky, Ph.D. thesis, Ecole Normale Supérieure, Paris, 1964.

[3] S. Haroche, Ph.D. thesis, Ecole Normale Supérieure, Paris, 1964.

[4] C. Audoin, C. Cohen-Tannoudji, S. Haroche, J.P. Schermann, Phys. Rev. Lett. 24861 (1970).

[5] C. Cohen-Tannoudji, S. Haroche, Phys. Rev. Lett. 24974 (1970).

[6] S. Haroche, S. Liberman, Frontiers in Laser Spectroscopy, edited by R. Balian (North Holland, Amsterdam, 1977 ), p. 1. 
[7] A. McLachlan, Proc. Royal Soc. London, Ser. A 271, 381 (1963).

[8] J.M. Wylie, J.E. Sipe, Phys. Rev. A 30, 1185 (1984).

[9] W. Jhe, K. Jang, Phys. Rev. A 53, 1126 (1996).

[10] R. Passante, F. Persico, Phys.Lett. A 200, 87 (1995).

[11] J.M.C. Malbouisson, B. Baseia, J. Mod. Opt. 46, 2015 (1999).

[12] L. Davidovitch, M. Brune, J. M. Raimond, S. Haroche, Phys. Rev. A 53, 1295 (1996).

[13] K. M. Fonseca-Romero, M. C. Nemes, J. G. Peixoto de Faria, A. N. Salgueiro, A. F. R. de Toledo Piza, Phys. Rev. A 58, 3205 (1998).

[14] N. Andion, A.P.C. Malbouisson, A. Mattos Neto, J. Phys. A 34, 3735 (2001).

[15] A.K. Likhoded, G.P. Pronko, Int. Journ. Theor. Phys. 36, 2335 (1997).

[16] W. Jhe, A. Anderson, E.A. Hinds, D. Meschede, L. Moi, S. Haroche, Phys. Rev. Lett. 58, 666 (1987).

[17] R.G. Hulet, E.S. Hilfer, D. Kleppner, Phys. Rev. Lett. 55, 2137 (1985).

[18] H. Morawitz, Phys. Rev. A 7, 1148 (1973)

[19] P. Milonni, P. Knight, Opt. Comm. 9, 119 (1973)

[20] D. Kleppner, Phys. Rev. Lett. 47, 233 (1981) 